\title{
La comunicación ante la trasversatilidad del género y el cambio climático
}

\author{
Susana Gómez Loperena ${ }^{1}$ \\ Guadalupe Alicia Cavazos Velázquez² \\ Martha Cecilia Manzano Ortiz ${ }^{3}$ \\ Universidad Autónoma de Tamaulipas
}

\section{RESUMEN}

Conocer cómo perciben e interpretan los mensajes comunicativos del cambio climático, los 94 hombres y mujeres estudiantes de Maestría en Comunicación de la Unidad Académica de Derecho y Ciencias Sociales de la Universidad Autónoma de Tamaulipas, en Ciudad Victoria, Tamaulipas, México. Estudio exploratorio cuantitativo y cualitativo con Etnometodología. Se encuestó a 52 mujeres y 42 hombres, en otoño de 2015 y primavera de 2016. Los hombres muestran un nivel más alto de percepción e interés en el cambio climático, avisos del pronóstico del clima y protección civil. Hombres y mujeres lo perciben como un fenómeno planetario con efectos lejanos. Internet como multiplataforma es el medio más usado.

Palabras clave: comunicación, género, cambio climático

\footnotetext{
${ }^{1}$ Maestra en Administración Pública, líder del Cuerpo Académico de Comunicación Humana y Tecnología, docente en la Maestría en Comunicación y la Licenciatura en Ciencias de la Comunicación, en la UADCS de la Universidad Autónoma de Tamaulipas, Ciudad Victoria. Correo electrónico: susana.loperena@gmail.com (autor corresponsal).

${ }^{2}$ Maestra en Comunicación Corporativa, docente en la Maestría en Comunicación y la Licenciatura en Ciencias de la Comunicación, en la UADCS de la Universidad Autónoma de Tamaulipas, Ciudad Victoria. Correo electrónico: gpeacavazosv@hotmail.com

${ }_{3}^{3}$ Maestra en Recursos Humanos, docente en la Licenciatura en Ciencias de la Comunicación en la UADCS de la Universidad Autónoma de Tamaulipas, Ciudad Victoria. Correo electrónico: mmanzano@uat.edu.mx

Este trabajo forma parte de la investigación "Evaluación de la comunicación interpersonal preferente de los jóvenes universitarios ante la brecha digital y de género y su efecto en la comprensión del cambio climático", realizada por integrantes del Cuerpo Académico en Formación de Comunicación Humana y Tecnología y financiada por el Programa de Fortalecimiento de Cuerpos Académicos, Convocatoria 2016, de la Secretaría de Educación Pública.
} 


\begin{abstract}
To know how the 94 men and women students of Communication Masters of the UADCS of the UAT, in Victoria, Tamaulipas, Mexico perceive and interpret the communicative messages of climate change. Quantitative and qualitative exploratory study with Ethnomethodology. 52 women and 42 men were surveyed; in the fall of 2015 and in the spring of 2016. Men show a higher level of perception and interest in climate change, weather forecasting and civil protection. Men and women perceive it as a planetary phenomenon with distant effects. Internet as a cross-platform is the media most used.
\end{abstract}

Keywords: communication, gender, climate change 


\section{INTRODUCCIÓN}

El cambio climático es un fenómeno donde la acción humana irrumpe en la naturaleza y altera su evolución natural lo que provoca fenómenos climáticos que producen riesgos y daños en el desarrollo cultural de las poblaciones, aunque todos los mexicanos viven las consecuencias del cambio climático, es aún un tema poco entendido, porque como señala Javier Urbina Soria, “...la única forma de conocer el cambio climático es recibiendo información de otras personas. (...) el conocimiento del cambio ambiental global en general y del cambio climático en particular depende de las acciones de comunicación que se emprendan" (Urbina y Martínez, 2006, p. 23).

Esta idea es reforzada por Boykoff quien expresa que "las representaciones mediáticas son un factor importante en el entendimiento y compromiso público de la ciencia climática y, por tanto, merecen una consideración crítica" (Boykoff, 2009, p. 119). "El papel de los medios de comunicación masivos en la construcción de las representaciones mediáticas de los problemas ambientales globales es (..) 'acentuar' o 'enfatizar' determinados aspectos de la realidad (...) a mayor énfasis en los medios de comunicación, mayor énfasis para el público" (Xambó, 1999, p. 448 en Meira Cartea, 2013, p. 53).
Es en la sociedad y los medios de comunicación donde se da "la presencia reiterada de pautas representacionales que parecen funcionar como estereotipos" (Meira Cartea, 2013, p. 53). La realidad se construye cognitivamente con percepciones, sensaciones recibidas por los sentidos e interpretadas en la mente en base a la información almacenada en esta. "La percepción involucra en su contenido, los valores, las tradiciones, los estereotipos, las vivencias y los conocimientos, que tienen los individuos sobre determinados aspectos o fenómenos de la vida" (Rodríguez y Álvarez, 2006, p. 1). Si los hombres y las mujeres son educados y expuestos a diferentes informaciones y experiencias de vida, el resultado será que sus procesos cognitivos a la hora de interpretar la realidad sean también diferentes. Henríquez, Mosqueda y Arias (2010, p. 66) citan a Koellinger y otros (2007), quienes señalan que "los hombres y las mujeres perciben e interpretan información diferente y que, como resultado, toman diferentes decisiones". Según Burin y Meler (1998) citados por Rocha y Díaz (2005, p. 48) "el género tiene carácter relacional, es decir, hombres y mujeres se configuran uno en función del otro, reconociendo lo que no se es versus lo que se debe ser y hacer y desarrollando lo que socialmente es permitido". 
"El género es una representación cultural, contiene ideas, prejuicios, valores, interpretaciones, normas, deberes, mandatos y prohibiciones sobre la vida de las mujeres y de los hombres" (Colas y Villaciervos, 2007, p. 37). El género también es influido por la respuesta al medio ambiente y los cambios climáticos en el contexto geográfico que se habita, así el clima determina los materiales para confeccionar la ropa, colores y estilos de arreglo personal, y los materiales empleados en edificaciones que usarán los seres humanos, así como la distribución de tareas entre mujeres y hombres dentro del núcleo familiar y en la sociedad.

El Banco Mundial dice que México es uno de los países más vulnerables por el fenómeno climático: 15\% de su territorio, 68\% de su población y 71\% de su economía están expuestos a las consecuencias adversas relacionadas con el clima (De Jong, López y Contreras, 2010). Estos datos nos confirman que más allá de las discusiones políticas o científicas sobre la veracidad o no del cambio climático, la realidad es que los fenómenos naturales climáticos afectan cada día a comunidades causando daños y pérdidas en vidas humanas, animales y vegetales, afectando infraestructuras y los recursos materiales y económicos. Es por ese motivo que se estudió como las muje-
Susana Gómez Loperena y otros

res y los hombres estudiantes de Maestría en Comunicación, que están siendo preparados para actuar como comunicadores participan en el proceso de la comunicación sobre el tema del cambio climático. Es dentro de la cultura mosaico actual, creada por los medios masivos de comunicación, el internet y las redes sociales, donde es necesario evaluar la función de los comunicadores en su papel de perceptores y futuros transmisores de mensajes mediáticos.

Las preguntas a responder atendiendo la brecha de género entre hombres y mujeres son:

-¿Cuál es el nivel de percepción e interés en el cambio climático, el pronóstico del tiempo y avisos de protección civil?

-¿Cómo definen con sus palabras el cambio climático las mujeres y los hombres?

-¿Cuáles son los medios de comunicación que utilizan para conocer del cambio climático?

-¿En qué medida envían y reciben información sobre cambio climático?

\section{DISEÑO DE INVESTIGACIÓN}

-Se realizó una investigación mixta cuantitativa y cualitativa en dos tiempos.

-Estudio exploratorio cuantitativo para describir los intereses y conductas comunica- 
tivas de mujeres y hombres sobre el cambio climático en estudiantes de la Maestría en Comunicación en la Unidad Académica de Derechos y Ciencias Sociales de la Universidad Autónoma de Tamaulipas. Se censó a la población de maestría con 94 estudiantes en total y se logró encuestar a 52 mujeres y 42 hombres. El cuestionario fue aplicado en los salones de clases de maestría en forma grupal durante los semestres de otoño de 2015 y primavera 2016, con una hora en promedio para ser contestado y con la asistencia de dos investigadoras para resolver dudas. El cuestionario es cerrado con opciones de respuesta dividido en seis secciones de la siguiente manera: 1. Datos generales (18 reactivos). 2. Apropiación de TIC, redes sociales, servicios de mensajería y videos (12 reactivos). 3. Uso académico de internet (cuatro reactivos). 4. Comunicación interpersonal (10 reactivos). 5 . Percepción de género (seis reactivos). 6. Percepción del cambio climático (nueve reactivos).

-Estudio cualitativo usando la Etnometodología para conocer el nivel de percepción e interés de mujeres y hombres sobre el cambio climático. "Los métodos cualitativos son los únicos que permiten percibir, medir y profundizar la valorización subjetiva que realizan las personas, grupos y comunidades humanas so- bre el sentido y significado" (Retamal, Rojas y Parra, 2011, p. 186). La Etnometodología fue desarrollada por Harold Garfinkel para investigar "las propiedades racionales de las expresiones contextuales y de otras acciones prácticas como logros continuos y contingentes de las prácticas ingeniosamente organizadas de la vida cotidiana” (Garfinkel, 2006, p. 20). Se siguió la propuesta de Garfinkel de pedir "a los estudiantes registrar conversaciones comunes, poniendo del lado izquierdo de la planilla lo que las partes efectivamente dijeron $y$, en el lado derecho, lo que ellos y sus compañeros entendieron de la conversación" (Garfinkel, 2006, p. 37). En agosto de 2016 se acudió al grupo de cuarto semestre de maestría con 18 alumnos (13 mujeres y cinco hombres), para aplicar un cuestionario abierto con cuatro preguntas: 1 . ¿Qué es para ti el cambio climático?, 2. ¿Qué fenómenos asocias con el cambio climático?, 3. ¿Cómo afecta tu vida diaria el cambio climático? y 4. ¿Qué acciones cotidianas realizas para prevenir el cambio climático? El propósito fue medir la percepción e interés sobre el tema. Para provocar la reflexividad en las respuestas se les pidió que respondiera las preguntas abiertas, se recogió el cuestionario y a la semana siguiente les fue regresado con la solicitud de que explican exactamente que quisieron decir con sus pala- 
bras, propiciando una experiencia disruptiva de su comunicación con la finalidad de descubrir los conocimiento y prácticas de la vida cotidiana asociadas al cambio climático desa- rrollados por las y los estudiantes de comunicación, a la tercera semana siguiente se les regresó el cuestionario con la solicitud de que aclararan sus respuestas. 


\section{RESULTADOS}

El Instituto Nacional de Estadística, Geografía e Información del Gobierno de México (Inegi) reporta en el 2015 que en Tamaulipas hay tres millones 441 mil 698 habitantes; de ellos el 49.2\% son hombres y el 50.8\% mujeres. De cada 100 tamaulipecos solo el 19.4\% concluye la educación superior (Inegi, “Cuéntame...”, 2015). Los estudiantes de la Maestría en Comunicación 2015-2016 son 94, 52 mujeres y 42 hombres. El rango de edad predominante es de 23 a 29 años con el 60\% de hombres y el 60\% de mujeres, los mayores de 30 años son 40\% de mujeres y 40\% de hombres. La mayoría son solteros, con el 70\% de hombres y $60 \%$ de mujeres; el 30\% de las mujeres y el $27 \%$ de los hombres están casados; el 3\% de los hombres vive en unión libre y el 6\% de las mujeres están divorciadas, $5.8 \%$ de las mujeres no respondió. Tienen hijos el $21 \%$ de las mujeres y el $40 \%$ de los hombres (ver cuadro 1).

\begin{tabular}{|c|c|c|c|c|c|c|c|c|c|c|c|c|c|c|}
\hline \multicolumn{15}{|c|}{$\begin{array}{l}\text { Cuadro 1. Edad, estado civil y número de hijos de mujeres y hombres } \\
\text { estudiantes de Maestría en Comunicación }\end{array}$} \\
\hline Edad & \multicolumn{2}{|l|}{ Sexo } & \multicolumn{2}{|l|}{ Soltero } & \multicolumn{2}{|l|}{ Casado } & \multicolumn{2}{|c|}{$\begin{array}{l}\text { Unión } \\
\text { libre }\end{array}$} & \multicolumn{2}{|c|}{ Divorciados } & \multicolumn{2}{|c|}{ Sibijos } & \multicolumn{2}{|c|}{ No bijos } \\
\hline AÑOS & $\mathrm{M}$ & $\mathrm{H}$ & $\mathrm{M}$ & $\mathrm{H}$ & $\mathrm{M}$ & $\mathrm{H}$ & $\mathrm{M}$ & $\mathrm{H}$ & $\mathrm{M}$ & $\mathrm{H}$ & $\mathrm{M}$ & $\mathrm{H}$ & $\mathrm{M}$ & $\mathrm{H}$ \\
\hline $23-29$ & $59.6 \%$ & $60 \%$ & $46.1 \%$ & $53 \%$ & $9.6 \%$ & $7 \%$ & $0 \%$ & $0 \%$ & $0 \%$ & $0 \%$ & $6 \%$ & $16 \%$ & $54 \%$ & $52.5 \%$ \\
\hline $30-39$ & $25 \%$ & $28 \%$ & $9.6 \%$ & $12 \%$ & $11.5 \%$ & $15 \%$ & $0 \%$ & $3 \%$ & $1.9 \%$ & $0 \%$ & $6 \%$ & $16 \%$ & $19 \%$ & $7 \%$ \\
\hline $40-56$ & $15.3 \%$ & $12 \%$ & $3.8 \%$ & $5 \%$ & $7.6 \%$ & $5 \%$ & $0 \%$ & $0 \%$ & $3.8 \%$ & $0 \%$ & $9 \%$ & $8 \%$ & $6 \%$ & $.5 \%$ \\
\hline Total & $100 \%$ & $100 \%$ & $59.6 \%$ & $70 \%$ & $28.8 \%$ & $27 \%$ & $0 \%$ & $3 \%$ & $5.7 \%$ & $0 \%$ & $21 \%$ & $40 \%$ & $79 \%$ & $60 \%$ \\
\hline \multicolumn{4}{|c|}{ Mujeres contestaron } & \multicolumn{2}{|c|}{$94.2 \%$} & \multicolumn{5}{|c|}{ Mujeres No contesto } & \multicolumn{2}{|l|}{$5.8 \%$} & \multicolumn{2}{|c|}{$100 \%$} \\
\hline \multicolumn{4}{|c|}{ Hombres contestaron } & \multicolumn{2}{|c|}{$100 \%$} & \multicolumn{5}{|c|}{ Hombres No contestaron } & \multicolumn{2}{|l|}{$0 \%$} & \multicolumn{2}{|c|}{$100 \%$} \\
\hline
\end{tabular}


Son estudiantes de tiempo completo el $55.7 \%$ de las mujeres y el $52.5 \%$ de los hombres, estudian y trabajan en $38.5 \%$ de las mujeres y el $47.5 \%$ de los hombres, $5.8 \%$ de las mujeres no contesto. Solo el $21.15 \%$ de las mujeres reporta ser ama de casa y el $2.5 \%$ de los hombres realizar tareas domésticas (ver cuadro 2).

\begin{tabular}{|l|l|l|l|l|l|}
\hline \multicolumn{2}{|c|}{ Cuadro 2. Actividades de mujeres y hombres estudiantes de Maestría en Comunicación } \\
\hline Tipo de estudiante & $M$ & $H$ & Ama de casa & $M$ & $H$ \\
\hline Estudiante de tiempo completo & $55.7 \%$ & $52.5 \%$ & No & $60 \%$ & $97.5 \%$ \\
\hline Estudia y trabaja & $38.5 \%$ & $47.5 \%$ & Sí & $21 \%$ & $2.5 \%$ \\
\hline No contestó & $5.8 \%$ & $0 \%$ & No contestó & $19 \%$ & $0 \%$ \\
\hline Total & $100 \%$ & $100 \%$ & Total & $100 \%$ & $100 \%$ \\
\hline
\end{tabular}


"Al identificar y entender las diferencias en la vulnerabilidad de las mujeres y los hombres ante el cambio climático se podrán desarrollar políticas que respondan al problema de la vulnerabilidad" (CINU, 2008, pp. 1-2). El cambio climático no afecta de la misma manera a hombres y mujeres, las mismas brechas sociales en la dotación de acceso a recursos de todo tipo, participación en procesos de toma de decisiones y distribución del trabajo remunerado y doméstico, que producen un desbalance en detrimento de la calidad de vida de las mujeres, se reflejan en las situaciones vivenciales ante fenómenos del cambio climático. "La perspectiva de género es prioritaria para planear las acciones de respuesta al cambio climático. El efecto del cambio climático sobre la igualdad de género es una amenaza constante que de no atenderse puede revertir los avances logrados" (Semarnat-INE, 2011, p. 14).

Mujer 34 años. Al principio asocié el cambio climático con problema y ahora lo asocio con importancia y necesidad urgente de cuidar los cambios climáticos y nuestro planeta para que no nos veamos muy afectados.

Hombre 26 años. No dejar luces prendidas, reciclar, usar más productos biodegradables y no desperdiciar agua.
En los resultados cuantitativos de la investigación se encontró que existe un nivel de percepción e interés en el cambio climático en los hombres del 82.5\%. "Cambios en el clima, en las estaciones del año, daños al ecosistema", "Al momento no percibo efecto mayor en mi vida diaria". El 12.5\% de los hombres dice no tener percepción ni interés "desconozco del tema", el $5 \%$ de los hombres no contestó. En las mujeres el 69\% muestra un nivel de percepción e interés por el cambio climático "El cambio climático es transformación que se ha dado al curso natural del clima y medio ambiente ha resultado y consecuencia de contaminación ambiental". El 25\% de las mujeres no muestran interés en el tema, pero sí señala percibirlo mediáticamente "son fenómenos que antes no veía y que ahora me doy cuenta que suceden gracias a los medios masivos de comunicación". El 6\% de las mujeres no contestó.

\section{PERCEPCIÓN E INTERÉS EN EL PRONÓSTICO DEL CLIMA}

"Diariamente, en los medios de información (radio, televisión, periódico) se anuncian los pronósticos del estado del tiempo, esto es, las posibles variaciones en las condiciones de clima esperado" (Conde, 2006, p. 6). Los informes sobre el pronóstico del clima son los

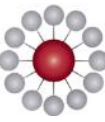


mensajes comunicativos más cercanos y cotidianos sobre el cambio climático al que tiene acceso la población. "Para ello es conveniente crear situaciones educativas en las que el sujeto-observador o contenedor de información pase a ser sujeto-intérprete y sujeto-actor social" (González y Meira, 2009, p. 29). Es decir, no basta con saber la situación climática que se aproxima sino también recibir información sobre medidas preventivas que es necesario tomar para protegerse de los efectos climáticos.

Los resultados cuantitativos muestran que el $82.5 \%$ de los hombres buscan mensajes sobre el pronóstico del clima: "la zona geográfica en donde vivo es muy caliente, por lo tanto, estas temperaturas afectan mi estado de ánimo y disminuyen $\mathrm{mi}$ rendimiento en las actividades que desarrollo". Al 12.5\% de los hombres no le interesa el pronóstico del clima (“que no pongo mucha atención") y el 5\% no contestó.

Las mujeres muestran en el 77\% percepción e interés en el pronóstico del clima ("asocio al cambio climático los eventos meteorológicos"), el 4\% de las mujeres dice no tener interés en el pronóstico del clima y el $19 \%$ no contestó la pregunta. 


\section{PERCEPCIÓN E INTERÉS EN AVISOS DE PROTECCIÓN CIVIL}

El Programa Nacional de Protección Civil 2014-2018, en el diagnóstico de la situación de México ante desastres naturales o accidentes provocados por acción humana, señala que: "La fuerza de la naturaleza se manifiesta a través de fenómenos perturbadores tales como sismos, volcanes, ciclones tropicales, inundaciones, etc.”. En otro aspecto del diagnóstico se reconoce que la población mexicana tiene poca información sobre el tema y que eso reduce la participación ciudadana:

La participación social en el Sistema Nacional de Protección Civil ha sido mínima, dada la escasa promoción a la cultura de protección civil. La población vulnerable y expuesta a un peligro cuenta con poca información sobre la situación de riesgo que vive, limitando su participación en la gestión del riesgo y su capacidad de resiliencia es mínima (DOF, 2014/04/30).

Alfabetizar a la población sobre cuestiones del cambio climático y estrategias de protección civil es una tarea aún en construcción donde participan actores de muy diversa índole, así González y Meira consideran que "Para facilitar este cambio cultural será necesaria una aplicación coordinada y complementaria de instrumentos educativos y de comunicación con otros de carácter normativo y coercitivo, además de cambios importantes en las políticas estructurales" (González y Meira, 2009, p. 35). "Por alfabetización ambiental entendemos la adquisición de información en el marco de un sustrato político y ético e implica una práctica social crítica que remite a la noción de ciudadanía" (González y Meira, 2009, p. 7-8). "El factor social en consecuencia, la forma en que la ciudadanía entiende y representa colectivamente y estructura su posición crítica ante el CC, es altamente relevante como componente estratégico si se quiere evitar los futuros escenarios que la ciencia pronostica” (Fussel, 2007; Stern, 2006; en Gumucio e Rau, 2012, p. 197).

Los resultados cuantitativos de la investigación indican que el interés por los avisos de protección civil es del 85\% en los hombres y del $67.3 \%$ en las mujeres, en cambio el 11.5\% de las mujeres y el $12.5 \%$ de los hombres no tienen interés en monitorear los avisos de protección civil. El 21.2\% de las mujeres y el 2.5\% de los hombres, no contesto. Lo cual muestra que casi una quinta parte de las mujeres estudiadas no perciben la existencia de riesgos por fenómenos del cambio climático (ver cuadro 3). 


\begin{tabular}{|l|l|l|l|l|l|l|l|l|l|}
\hline \multicolumn{7}{|c|}{ Cuadro 3. Nivel de percepción e interés de mujeres y hombres en cambios climático, } \\
pronóstico del clima y avisos de protección civil \\
\hline \\
\end{tabular}




\section{PERCEPCIÓN E INTERPRETACIÓN DEL CAMBIO CLIMÁTICO}

La vida cotidiana de mujeres y hombres es atravesada por los mensajes mediáticos emitidos por los medios masivos de comunicación locales, nacionales y globales además de aquellos mensajes emitidos por los "prosumidores" usuarios activos en las redes sociales que comunican sus percepciones, emociones y opiniones sobre el acontecer diario en sus relaciones familiares, laborales, escolares, sociales y de contacto con fenómenos naturales del cambio climático o creados por la acción humana que se traducen en sorpresa, riesgo y vulnerabilidad. Piñuel señala que existe "una cotidianeidad que conduce a la producción y reproducción de discursos, determinados tanto por los MCS masivos como por la relación de sus mensajes con repertorios y esquemas mentales y de acción propios" (Piñuel, 2013, p. 29, 30).

En lo referente al discurso políticoadministrativo la Convención Marco de las Naciones Unidas sobre el Cambio Climático en su Artículo 1. Define al cambio climático como "un cambio de clima atribuido directa o indirectamente a la actividad humana que altera la composición de la atmosfera mundial y que se suma a la variabilidad natural del clima observada durante períodos de tiempo com- parables" (UNFCCC, 1992, p. 4). Esta variabilidad en el clima muchas veces pasa inadvertida por mujeres y hombres ocupados en sus tareas diarias sobre todos los que habitan zonas urbanas quienes consideran al cambio climático un asunto de científicos y difícil de entender, los medios masivos de comunicación han construido imágenes del cambio climático en zonas geográficas distantes de las ciudades lo que ha provocado como dice Oltra y otros, que: "La mayoría de la población tiende a asociar el cambio climático con fenómenos lejanos como el deshielo, los incrementos genéricos en la temperatura o diferentes problemas medioambientales" (Oltra y otros, 2009, p. 7). Urbina considera la importancia de estudiar las percepciones y conductas de los mexicanos sobre los conocimientos que estos poseen sobre el fenómeno y las actitudes hacia causas y efectos (Urbina, 2006, p. $72,73)$. Agrega la existencia de cinco características de orden psicológico que complican el fenómeno: "La baja visibilidad del cambio global, La extrema dilación en mostrar la relación causa-efecto, La psicofísica de los eventos de baja probabilidad, La distancia social entre actores y víctimas del cambio ambiental, El bajo índice subjetivo de costo/efectividad" (Urbina, 2006, p. 67-68). Se usará esta clasificación para el análisis de respuestas a las pre- 
guntas abiertas de: ¿Qué es para ti el cambio climático?, ¿Qué fenómenos asocias con el cambio climático?, ¿Cómo afecta tu vida diaria el cambio climático? y ¿Qué acciones cotidianas realizas para prevenir el cambio climático?

-Ejemplo: baja visibilidad del cambio global. "Los cambios de temperatura diarios, estacionales o regionales, sus umbrales que no son fáciles de detectar y no pueden procesarse directamente en la percepción humana" (Urbina, 2006, p. 67).

-Testimonio 1. Mujer de 27 años, estudia y trabaja, es soltera, localidad urbana. Tiene computadora, tablet, laptop, celular, servicio móvil y fijo de internet en casa y trabajo.

-¿Qué es el cambio climático? Son los cambios meteorológicos que sufre el medio ambiente. Los cambios climáticos que ocurren día a día y a toda hora, aunque a veces no sean muy notorios. El clima del medio. Pues todo, porque afecta en la rutina diaria. Los cambios en el estado del tiempo. Son parte del día a día. No hay manera en que los cambios climáticos no afecten el día a día de cada persona. Afectan para bien o para mal, la vida diaria.

-Ejemplo: extrema dilación en mostrar la relación causa-efecto. "Es extremadamente largo el rango temporal para conectar las acciones humanas y sus consecuencias" (Urbina, 2006, p. 68).

-Testimonio 2. Hombre de 31 años, estudiante de tiempo completo, soltero, localidad urbana. Tiene computadora, tablet, laptop, celular, servicio de internet móvil y fijo en casa, escuela y trabajo.

-¿Qué es el cambio climático? No he definido mi criterio con respecto al tema. Creo que por un lado el uso indiscriminado de los recursos naturales ha causado estragos en el planeta. Pero por el otro lado creo también que los cambios en el clima han existido siempre en el planeta y son cíclicos en cada época del planeta tierra. Esto ha sido aprovechado por una agenda global que pretende transformar el uso de las energías para beneficio de intereses particulares. De alguna manera he recibido información ambigua con cierto toque de conspiración. Probablemente tiene que ver con la información que me interesa leer. Que no he entendido el tema al 100\%. No me he interesado por el tema lo suficiente.

-Ejemplo: psicofísica de los eventos de baja probabilidad. "Las personas tienden a subestimar la frecuencia relativa de eventos raros, aun cuando la frecuencia absoluta incremente" (Urbina, 2006, p. 68). 
-Testimonio 3. Mujer de 34 años, estudiante de tiempo completo, casada, con un hijo, ama de casa, localidad rural. Tiene computadora, tablet, laptop, servicio de internet móvil y fijo en casa.

-¿Qué es el cambio climático? Es un problema ambiental si no es normal el calentamiento global. Qué es un cambio en la distribución meteorológica, el cual puede ser un problema a largo plazo si no cuidamos nuestro planeta. Derretimiento de hielo en zonas glaciales, olas de calor, animales en riesgo de extinción. Afecta a todo el planeta es mi casa y se está deteriorando en general. Trato de ser amigable con el medio ambiente.

-Ejemplo: distancia social entre actores y victimas del cambio ambiental. "El aprendizaje social humano tiende a ser una función de la proximidad interpersonal de los participantes, mientras que el cambio ambiental opera en distancias espaciales y temporales muy grandes" (Urbina, 2006, p. 68).

-Testimonio 4. Mujer de 35 años, estudia y trabaja, soltera. Tiene computadora, laptop y celular.

-¿Qué es el cambio climático? Los desajustes relacionados con la cuestión climatológica. Que, dentro del concepto de cambio climático, agrupa diversos desajustes y/o cambios ambientales. Lo que entiendo es que algunos cambios son hasta cierto punto naturales, es decir, propio de la vida de la tierra, y por otro lado algunos desastres son generados por los desechos tóxicos de empresas, uso de productos dañinos, se dice que el CERN en Suiza, cada vez que está en uso, provoca que se generen grandes tormentas en diferentes lugares, etc. La tala de árboles y el no plantar y dar seguimiento afecta en lo particular a la calidad del aire, smog, en que no existe cultura sobre el arreglo de los autos para que no expidan gas que es sumamente dañino, al igual que cultura en la separación de basura, desechos tóxicos como pilas, etc. Al parecer existen dos teorías acerca del tema. El primero habla de que el cambio climático es causado por el hombre. El segundo es que solamente son procesos naturales "normales" hasta cierto punto y es real. Mi opinión es que la tierra está sustentada y es muy vasta y con gran riqueza, hay lugar para mucha gente (contrariamente a las medidas que se están tomando sobre reducción de la población por medios poco éticos). Por otra parte, el ser humano genera grandes materiales tóxicos, no existe respeto por los seres humanos, ni por los animales, ni por las plantas, etc. Hasta cierto punto el humano tiene responsabilidad ante esto, pero pienso que los cambios que se dan son naturales, con excepción de las 
cuestiones donde el ser humano experimenta (bombas nucleares, el CERN, etc.), que sí afectan (deshielos, cuestiones volcánicas, solares, etc.). Pues que vivo en este planeta y soy parte de.

-Ejemplo: bajo indice subjetivo de costo/ efectividad de la conducta protectora del ambiente. "Los sujetos no perciben efectos inmediatos o importantes del cambio en su comportamiento" (Urbina, 2006, p. 68).

-Testimonio 5. Hombre de 26 años, estudiante de tiempo completo, soltero, localidad urbana. Tiene computadora, laptop, celular, servicio de internet móvil y fijo en escuela y trabajo.

-¿Qué es el cambio climático? Cambios abruptos al ecosistema donde se generan cambios en las estaciones del año y por lo tanto cambios en las temperaturas. Cambios que se han venido generando a consecuencia del calentamiento global, como por ejemplo el derretimiento de los glaciares, los fuertes cambios en las estaciones del año. Que se están generando cambios de diferentes tipos en nuestro planeta, fuertes lluvias, inundaciones, incendios forestales, altas y bajas temperaturas. Me afecta en mi salud ya que por los fuertes calores requiero tomar más agua o puedo deshidratarme, puedo llegar a contraer cáncer de piel por los fuertes rayos de sol. La gran cantidad de contaminación que se ha acumulado a lo largo de los años ha ocasionado grandes cambios en el ecosistema, lo cual ha generado daños irreversibles a nuestro planeta. Los cambios que se han hecho notar han sido la consecuencia de la contaminación que la humanidad ha causado. Afecta mis actividades y mi salud.

\section{LOS MEDIOS DE COMUNICACIÓN QUE UTILIZAN PARA CONOCER SOBRE EL CAMBIO CLIMÁTICO}

Díaz (2009) propone la necesidad de realizar estudios de comunicación orientados a investigar la brecha entre la expresión discursiva de actores del cambio climático como son científicos, trabajadores de los medios de comunicación y políticos, pues expresa que: "la construcción de imaginarios culturales comprometidos con el medioambiente, compete a la comunicación más allá del análisis del tratamiento que los medios prestan a los problemas del cambio climático y el calentamiento global" (Díaz, 2009, p. 15).

"Piñuel Raigada hace una pregunta básica: cómo evaluar, diseñar y realizar la mejor comunicación posible sobre el riesgo de que se agrave aún más el proceso ya irreversible del calentamiento global del planeta, con to- 
das las consecuencias que de ello se derivan" (Fernández y Mancinas, 2013, p. 18). Esta pregunta compromete el trabajo de los docentes del área de Comunicación y de las instituciones que ofertan licenciaturas, maestrías y doctorados en Comunicación, sobre qué acciones se llevan a cabo para preparar a los futuros comunicólogos ante esta necesidad comunicativa de la sociedad. Fernández cita a “el periodista científico Manuel Calvo (...) los medios de comunicación están llamados a realizar, junto con el sistema educativo, un esfuerzo gigantesco de instrucción prospectiva, y en una escala sin precedentes hasta ahora” (Fernández y Mancinas, 2013, p. 12) con el objetivo de difundir el fenómeno del cambio climático y la cultura de la protección civil.

Radica aquí la importancia de monitorear como están interpretando el fenómeno del cambio climático los futuros comunicólogos que tendrán el papel de construir la información mediática sobre el asunto, ya que "es importante considerar la función de los medios de comunicación en la ciencia, política y practica climática actuales, y examinar tanto los motivos que guían las representaciones mediáticas como los efectos de las mismas" (Boykoff, 2009, p. 119).
Susana Gómez Loperena y otros

Mujer 26 años. Que gracias a los medios de comunicación sabemos cómo reaccionar ante fenómenos raros. Sé que va a pasar gracias a los medios. Los medios ayudan a prevenirse cuando suceden estos fenómenos.

Hombre 26 años. He recibido información de diversas fuentes.

Los datos cuantitativos de la investigación señalan que el internet es la multiplataforma mediática utilizadas por el $61.5 \%$ de las mujeres y el $47 \%$ de los hombres para adquirir datos sobre el cambio climático ("Tal vez he visto demasiadas películas"); en segundo lugar está la mezcla de internet y televisión con el $11.5 \%$ de las mujeres y el $7 \%$ de los hombres; en tercer lugar, la mezcla internet, televisión y radio, con el $4 \%$ en mujeres y $7 \%$ en hombres; internet y radio es usado por el $2 \%$ de los hombres y prensa e internet por el $2 \%$ de las mujeres. Con respeto al uso de medios de comunicación tradicionales, la televisión se usa en el $6 \%$ de las mujeres y la prensa en el $2 \%$ de las mujeres.

Todos los medios son usados por el $11.5 \%$ de las mujeres y el $4 \%$ de los hombres. El 2\% de las mujeres no respondió y el 33\% de los hombres tampoco ("Tal vez no hay interés en el tema"). 
RECIBO Y ENVÍO INFORMACIÓN SOBRE CAMBIO CLIMÁTICO

Independientemente de las políticas públicas para atender el cambio climático esta la forma como la población se acerca al asunto, Isaac Márquez en 2016, cita a Lezama quien trata este tema y especifica que:

La manera como la sociedad percibe o valora los problemas ambientales no depende básicamente de los riesgos que representa o de la veracidad de su existencia, sino de la manera como son internalizados por la sociedad en los ámbitos normativos, cognoscitivos y simbólicos (Lezama, 2008; en Márquez, 2016, p. 3).

Los datos cuantitativos de la investigación muestran que reciben información sobre cambio climático el $23 \%$ de las mujeres y el 35 $\%$ de los hombres, envían información el 25\% de las mujeres y el $27.5 \%$ de los hombres a sus contactos en las redes sociales. Realizan ambas funciones el 15\% de las mujeres y el $37.5 \%$ de los hombres. No contestó el 36\% de las mujeres y el $32.5 \%$ de los hombres.

Mujer 35 años. Que la calidad de vida que tengan nuestros hijos depende de las acciones que realicemos hoy. Que nuestras acciones deben ser encaminadas a prevenir y cuidar nuestro planeta.

\section{CONCLUSIÓN}

$\mathrm{Al}$ estudiar a futuros comunicólogos estudiantes de la Maestría en Comunicación de la UADCS de la UAT, se encontró que son en su mayoría menores de 30 años, solteros y estudiantes de tiempo completo, habitan zonas urbanas y cuentan con computadora, tablet, laptop, celular y servicio móvil y fijo de internet. Los hombres muestran mayor percepción e interés en el fenómeno del cambio climático, y están más atentos al pronóstico del tiempo y a los avisos de protección civil que las mujeres. Para las mujeres el cambio climático es parte de la rutina diaria que afecta sus tareas cotidianas, sobre todo, por el calentamiento global que consideran un fenómeno natural que está siendo afectado por el ser humano con acciones como: la tala de árboles, el daño a la calidad del aire por vehículos, la falta de cultura en el tratamiento de la basura y desechos tóxicos; lo que provoca enfermedades y daña la flora y fauna. Las mujeres buscan ser amigables con el medio ambiente, consideran que el ser humano es responsable del cuidado del planeta. Para los hombres el cambio climático es un asunto difícil de entender, relacionado con el uso indiscriminado de recursos naturales y el uso de energías para fines particulares, lo que provoca un calentamiento global que afecta las estaciones del año

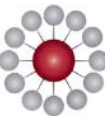


y provoca enfermedades por la contaminación. El uso de la multiplataforma de internet predomina tanto en mujeres como en hombres para acceder a información sobre el cambio climático; muy por debajo está el uso de los medios masivos de comunicación tradicionales. Los hombres reciben mayor contenido informativo sobre cambio climático que las mujeres, sin embargo, la proporción en que hombres y mujeres envían información sobre cambio climático a sus contactos en las redes sociales es similar. Los hombres son más activos en este tema informativo que las mujeres. Para las mujeres el cambio climático se relaciona con cambios meteorológicos, desechos industriales, deforestación, contaminación y los ciclos de cambios naturales del planeta. Para los hombres el cambio climático se rela- ciona con el uso de las energías. Las mujeres asocian el cambio climático con el derretimiento del hielo glaciar, olas de calor, extinción de animales, actividad volcánica y tormentas solares. Los hombres asocian el cambio climático con fuertes lluvias, inundaciones, incendios forestales y temperaturas extremas. Tanto hombres como mujeres consideran que el cambio climático afecta su salud y su rutina de vida diaria. Sin embargo, no mencionan acciones para prevenir el cambio climático. Se observa falta de conocimientos sobre cambio climático y desinterés en el tema en la población estudiada, motivo por el cual se recomienda introducir materias sobre divulgación de la ciencia del cambio climático en los programas curriculares de las Ciencias de la Comunicación en todos sus niveles. 


\section{FUENTES CONSULTADAS}

Boykoff, T. (2009). "Los medios y la comunicación científica. El caso del cambio climático", En: INFOAMERICA: Iberoamerican Communication Review, núm. 1, 117-120. Recuperado el 20 de febrero del 2017 de https://dialnet.unirioja.es/servlet/articulo?codigo=3910914.

Colas, P. y Villaciervos, P. (2007). "La interiorización de los estereotipos de género en jóvenes y adolescentes”. En: Revista de Investigación Educativa, vol. 25, núm. 1, 35-58. Recuperado el 10 de enero del 2017 de revistas.um.es/rie/article/viewFile/96421/92631.

Conde C. (2006). México y el cambio climático global, 1-28, México, DF: UNAM. Recuperado el 2 de marzo del 2017

de http://www.atmosfera.unam.mx/editorial/libros/mexico_cambio_climatico/Mexico_y_el_cambi o_climatico_global.pdf.

Díaz Nosty, B. (2009). "Cambio climático, consenso científico y construcción mediática. Los paradigmas de la comunicación para la sostenibilidad”. En: Revista Latina de Comunicación Social, núm. 64, 99-119. La Laguna (Tenerife): Universidad de La Laguna. Recuperado el 25 de abril del 2017 de http://www.revistalatinacs.org/09/art/09_808_15_climatico/latina_art808.pdf.

Diario Oficial de la Federación, DOF (30/04/2014). "Programa Nacional de Protección Civil 2014-2018". Recuperado el 15 de marzo del 2017 de http://www.dof.gob.mx/nota_detalle.php?codigo $=5343076 \&$ fecha $=30 / 04 / 2014$.

De Jong F., López A. y Contreras, L. (2010). "Cambio climático: los retos y las oportunidades para México”. En: Revista Expansión (19/10/2010). Recuperado el 24 de marzo del 2016 de expansion.mx/estilo/2016/03/24/cambio-climatico-los-retos-y-las-oportunidades-para-mexico.

Fernández, R. (director) y Mancinas-Chávez, R. (coordinadora) (2013). Actas de las Jornadas Internacionales Medios de Comunicación y Cambio Climático, 1-398. Sevilla: Fénix Editora. Recuperado el 2 de mayo del 2016 de https://idus.us.es/xmlui/bitstream/handle/11441/30820/ActasJornadasMediosdeComunicacion. pdf?sequence $=1$.

Garfinkel, H. (2006). Estudios en Etnometodologia. Centro de Investigaciones Interdisciplinarias en Ciencias y Humanidades. Bogotá: Universidad Nacional de Colombia, XI, 1-331. Colección Autores, Textos y 
Temas. Ciencias Sociales, núm. 52. Recuperado el 9 de marzo del 2017 de https://docs.google.com/file/d/0B5UfjjAP0C2FQnlUWWI2OEd5V3M/edit.

González, E. y Meira, P. (2009). "Educación, comunicación y cambio climático. Resistencias para la acción social responsable”. En: Revista Trayectorias, vol. 11 núm. 29, julio-diciembre, 6-38. Recuperado el 4 de abril del 2017 de www.pincc.unam.mx/reducc/actividades_2016/gonzalez.pdf.

Grupo Interagencial de Género del Sistema de las Naciones Unidas en México (2008). Ficha Informativa sobre Género y Desarrollo, núm. 7, 1-2. Recuperado el 29 de junio de 2017 de www.cinu.org.mx/gig/Documentos/ambiente.pdf.

Henríquez, M. C., Mosqueda, C. E. y Arias, A. (2010). "La creación de empresas en Colombia desde las percepciones femenina y masculina". En: Economía Gestión y Desarrollo. Cali (Colombia), núm. 10, diciembre, 61-77. Recuperado el 3 de marzo del 2017 de https://core.ac.uk/download/pdf/6552299.pdf.

Gumucio, C. y Muñoz Rau, J. (2012). "Elites universitarias y cambio climático”. En: Ambiente \& Sociedade, vol. 15, núm. 2, 195-218. Sao Paulo. Recuperado el 7 de febrero del 2017 de http://www.scielo.br/scielo.php?pid=S1414-753X2012000200011\&script=sci_arttext.

Instituto Nacional de Estadísticas Geografía e Información, Inegi (2015). "Cuéntame. Información por entidad Tamaulipas". Recuperado el 24 de febrero del 2017 de cuentame.inegi.org.mx $/$ monografias/informacion/Tam/Poblacion/default.aspx?tema=ME\&e=28.

Lezama, J. L. (2008). La construcción social y politica del medio ambiente. Ciudad de México: El Colegio de México.

Márquez, R. I. (2016). "Conocimientos y percepciones sobre el cambio climático en personal de la administración pública municipal del estado de Campeche". En: Revista Iberoamericana de Producción Académica y Gestión Educativa, núm. 4, enero-junio, 1-8. Recuperado el 15 de enero del 2017 de https://www.pag.org.mx/index.php/PAG/article/download/559/596.

Meira y Cartea, P. (2013). "Problemas ambientales globales y educación ambiental: una aproximación desde las representaciones sociales del cambio climático". En: Revista Integra Educativa, vol. 6, núm. 3, 29-64. Recuperado el 5 de mayo del 2017 de www.scielo.org.bo/scielo.php?script=sci_arttext\&pid=\$199740432013000300003\&lng=es\&tlng=es.

Naciones Unidas (1992). "Convención marco de las Naciones Unidas sobre el cambio climático". 1-26. Recuperado el 27 de febrero del 2017 de https://unfccc.int/resource/docs/convkp/convsp.pdf. 
Oltra Ch., Solà R., Sala, R., Prados, A. y Gamero, N. (2009). "Cambio climático: percepciones y discursos públicos”. En: Revista Prisma Social, núm. 2, junio, 1-23. Recuperado el 13 de marzo del 2017 de http://www.isdfundacion.org/publicaciones/revista/pdf/n2_9.pdf.

Piñuel, J. L. (2013). "El discurso hegemónico de los media sobre el cambio climático (riesgos, incertidumbre y conflicto) y estrategias de intervención". En: Actas de las Jornadas Internacionales Medios de Comunicación y Cambio Climático, 27-44. Recuperado el 24 de marzo del 2017 de https://idus.us.es/.../Pages\%20from\%20ActasJornadasMediosdeComunicacion-3.pdf?

Retamal, M. R., Rojas y Parra, J. O. (2011). "Percepción al cambio climático y a la gestión del agua: aportes de las estrategias metodológicas cualitativas para su comprensión”. En: Ambiente \& Sociedade, vol. 14, núm. 1, 175-194. Recuperado el 14 de abril del 2017 de http://www.scielo.br/scielo.php?pid=S1414-753X2011000100010\&script=sci_arttext.

Rocha, T. E. y Díaz, R. (2005). "Cultura de género: la brecha ideológica entre hombres y mujeres”. En: Anales de Psicología, vol. 2, núm. 1, junio, 42-49. Recuperado el 22 de febrero del 2017 de http://search.proquest.com/openview/1bc3a5c0ccccef558e2352f6ff5599a1/1?pqorigsite $=$ gscholar\&cbl $=1606360$.

Rodríguez, A. y Álvarez, L. (2006). "Percepciones y comportamiento de riesgo en la vida sexual y reproductiva de los adolescentes". En: Revista Cubana Salud Pública, núm. 32, 1-9. Recuperado el 2 de febrero del 2017 de bvs.sld.cu/revistas/spu/vol32_1_06/spu08106.htm.

Secretaría de Gobernación (2016). "Programa Nacional de Protección Civil 2014-2018”, 1-46. Recupe$\begin{array}{llllll}\text { rado el } & 28 & \text { de } & & \end{array}$ sct.gob.mx/fileadmin/ProteccionCivil/programaGeneralProteccionCivil.pdf.

Secretaria de Medio Ambiente y Recursos Naturales, Instituto Nacional de Ecología (2011). Voces ante el cambio climático. Ideas sustentables, México, DF: Ideas Sustentables, 1-21. Recuperado el 20 de enero del 2017 de http://www.semarnat.gob.mx/gobmx/biblioteca/biblio.html.

Urbina, J. y Martínez, J. (compiladores) (2006). Más allá del cambio climático. Las dimensiones psicosociales del cambio ambiental global, 1era. Edición, 1-228. México: Secretaría de Medio Ambiente y Recursos Naturales, Instituto Nacional de Ecología, Universidad Nacional Autónoma de México, Facultad de Psicología. Recuperado el 9 de abril del 2017 de http://biblioteca.semarnat.gob.mx/janium/Documentos/211895.pdf. 